Vietnam Journal of Mechanics, VAST, Vol. 42, No. 3 (2020), pp. $293-305$

DOI: https://doi.org/10.15625/0866-7136/15512

Dedicated to Professor J.N. Reddy on the Occasion of His $75^{\text {th }}$ Birthday

\title{
DYNAMIC CRACK PROPAGATION IN SPECIMENS WITH A SURFACE IRREGULARITY
}

\author{
Ali Hassanirad ${ }^{1}$, Vahid Vaziri ${ }^{1}$, Ko-Choong Woo ${ }^{2}$, Marian Wiercigroch ${ }^{1, *}$ \\ ${ }^{1}$ Centre for Applied Dynamics Research, School of Engineering, University of Aberdeen, Aberdeen AB24 3UE, UK \\ ${ }^{2}$ Department of Mechanical, Materials and Manufacturing Engineering, University of Nottingham Malaysia, \\ Semenyih, Selangor Darul Ehsan, Malaysia \\ *E-mail: m.wiercigroch@abdn.ac.uk
}

Received: 15 September 2020 / Published online: 27 September 2020

\begin{abstract}
Initiation of cracks and their propagation in prescribed orientations on tubular specimens has been made possible on a dynamic fatigue testing rig developed at the University of Aberdeen. This rig was originally designed to perform experiments on single edge notched beams (SENB) [1,2]. Modifications have recently been made so as to accommodate experimental tests on tubular specimens with a range of sizes and other cross sections. Crack initiation at grooves on such specimens has been followed by crack growth. At the same time, lateral oscillations of cracked specimen have been measured, as well as accelerations of base excitation, masses above and below cracked specimen. Forces on these two masses have been observed by two load cells attached at positions close to specimen. These load cells facilitated the measurement of stresses in experiment. Crack length time histories have also been constructed by applying an alternating current potential difference (ACPD) method. Fatigue cracks were initiated at the pre-cut grooves in aluminium tubular specimens. Three specimens with different groove sizes were tested in fifteen individual experiments. A three-dimensional Finite Element model was established for each specimen so as to calculate the stress concentration factor (SCF). This formed the basis of determining the amplitude of forcing input made possible by an electromechanical shaker. The phase shifts of acquired time histories has provided some indication of energy transfer mechanism during fatigue and system dynamic response. Observations of cracked specimen displacement during experiments was compared against calculated displacement from elastic theory. Nonlinear responses were observed, suggesting nonlinear stiffness characteristics of the specimen due to discontinuities introduced by crack growth and plasticity effects. Comparison of damage combinations in experimental observation was made with predictions from BS EN 1999-1-3. All experimental observations of total damage combination were higher than predicted values by the latter code.
\end{abstract}

Keywords: fatigue crack, dynamics loading, FEM, experiments.

\section{INTRODUCTION}

Estimation of fatigue life of mechanical or structural components under cyclic and random loading is a key issue in the design of mechanical components in machinery or assessment of offshore structures for suitability of the service to ensure adequate fatigue life. Design standards and codes such as DNVGLRP-C203 [3] are updated regularly to reflect the advances and findings in the field of fatigue tests and fracture mechanics, ensuring code-compliant fatigue life in structures, such as offshore jackets and wind turbine structures.

Accurate prediction of fatigue life can save costs by extending the life of an offshore asset so that targeted subsea inspections are less frequent. Fatigue experiments can minimise the safety factors and the closer the experiment condition is to the real condition the better the fatigue life estimation would be. The S-N curves in the codes are typically conservative and specific fatigue experiments can result in developing more refined and accurate S-N curves. 
A motivation behind this research is to enable fatigue testing on tubular specimens and increase the accuracy of the real life fatigue testing. Tubular sections are the most common sections used in making the offshore structural jacket members such as legs and braces. A number of current researches focus of on fatigue performance of pipe sections in different usages such as in pipelines [4], caissons [5] and risers [6]. The cyclic loads on pipe elements induced by wave, current and wind cause fatigue damage and the design for fatigue and prediction of the life is of paramount importance. Tubular specimens with different causes of stress raisers such as groove or hole can be tested using the proposed experimental fatigue rig.

Demonstrating higher fatigue life or improve fatigue performance of metals can be done through standard mechanical process in practice such as peening or toe grinding in welds or innovative methods like surface rolling treatment as studied by Carneiro et al. [7]. In the current study the aim is to minimise uncertainties in fatigue experiments with monitoring loads, displacements and crack propagation which can result in optimum fatigue design. The use of aluminium alloys in structural engineering is increasing due to the light weight and high corrosion resistance properties. Xiang et al. [8] studied 6061-T6 aluminium alloy under ultra-low cycle fatigue scenario which is mainly practical in use of aluminium in seismic prone regions. This study focuses on low and high cycle fatigue which covers a wider range industry usage.

Fatigue starts with nucleation of microcracks followed by coalescence and growth of microcracks [9]. The final stages involve long crack growth and final fracture. Crack start at high stress concentration location, which is normally in the region of geometry change, welding, connection points, inclusions, porosity, defects or discontinuities.

New fatigue rigs are being developed to perform fatigue experiments for practical purposes such as the fatigue rig developed by Okeke et al. [10] or Nagabhooshanam et al. [11]. Although, none of these rigs enable direct measurement of forces applied on the specimens during the course of fatigue experiments. Many fatigue experiment use servohydraulic machines such as the experiment carried out by Wei et al. [12]. Foong et al. [1] designed a novel fatigue rig in the University of Aberdeen and later studied parameter identification [2]. This rig was used in subsequent research on nonlinear vibration caused by fatigue [13] and chaos caused by fatigue crack growth [14].

This fatigue rig enables fatigue experiments on specimens under bending. The modifications allow the rig to perform tests on different section sizes while monitoring the exact dynamic force applied to the specimens using newly designed load cells. In this research ACPD technique was used to monitor the initiation and propagation of the crack. This technique was also used in fatigue experiment researches such as [15] and [16]. Mikulski et al. [17] showed that the ACPD system is sensitive to early crack growth. Measuring the crack in the experiment without interruption during the test is one of the advantages of ACPD system.

The main purpose of the present work is to modify and enhance the existing fatigue rig which can be used to perform fatigue experiments on different sections including pipe sections. The structure of this paper is as follows. Section 2 describes the experimental setup used for the fatigue rig, describing the original design and current modifications which includes introducing two new load cells, plastic rollers and sliding flexible jaws to accommodate wide range of section sizes and shapes. Then we describe the instrumentation and data acquisition. The calculation of system mechanical parameters and calibration of load cells, ACPD, and Eddy current proximity sensors are detailed. Next the properties of tubular sections are presented followed by the finite element (FE) model of specimens to derive the stress concentration factors (SCF). Experimental results are presented in Section 3. Harmonic test results are demonstrated initially and then results of fifteen fatigue experiments including achieved number of cycles and crack growth from ACPD reading in the fatigue experiments are presented. The monitored displacement is compared against the calculated elastic displacement to identify the transition from elastic to nonlinear as a result of crack formation in the specimen and change of stiffness. Finally, the conclusion and discussion of the main results and suggestions for future research are presented in Section 4 . 


\section{EXPERIMENTAL SETUP}

To facilitate the experimental testing of tubular specimens which have surface discontinuities, modifications to an existing experimental rig are described, which enable this geometry to be held securely. Specific information pertaining to the size and geometry of specimen are provided, as well as the irregularity on the surface which induces stress concentration. The experimental setup is also described to include instrumentation and data acquisition system. The amplitude of forcing input was quantified by numerically solving the geometry of specimen in consideration in finite element analysis so as to compute stress concentration factors. These, in turn, allow for the calculation of force values, which constitute an input to the cracked specimens during the experiment.

\subsection{Rig description}

The schematic diagram of the modified fatigue rig is demonstrated in Fig. 1. The dynamic fatigue testing rig which was developed at the University of Aberdeen has gone through major design modifications. This rig was originally designed to perform experiments on the single edge notched beam (SENB) while put on the shaker [1,2]. Signal inputs to the shaker were implemented in Labview software. Electrical signals from accelerometers were acquired to measure the accelerations of mass above and below the specimen, as well as monitor the motions of the shaker table. At the same time, these were observed on oscilloscopes to ensure the integrity of acquired data. Electrical noise from a proximity sensor was filtered so as to quantify the vibrations of cracked specimen.
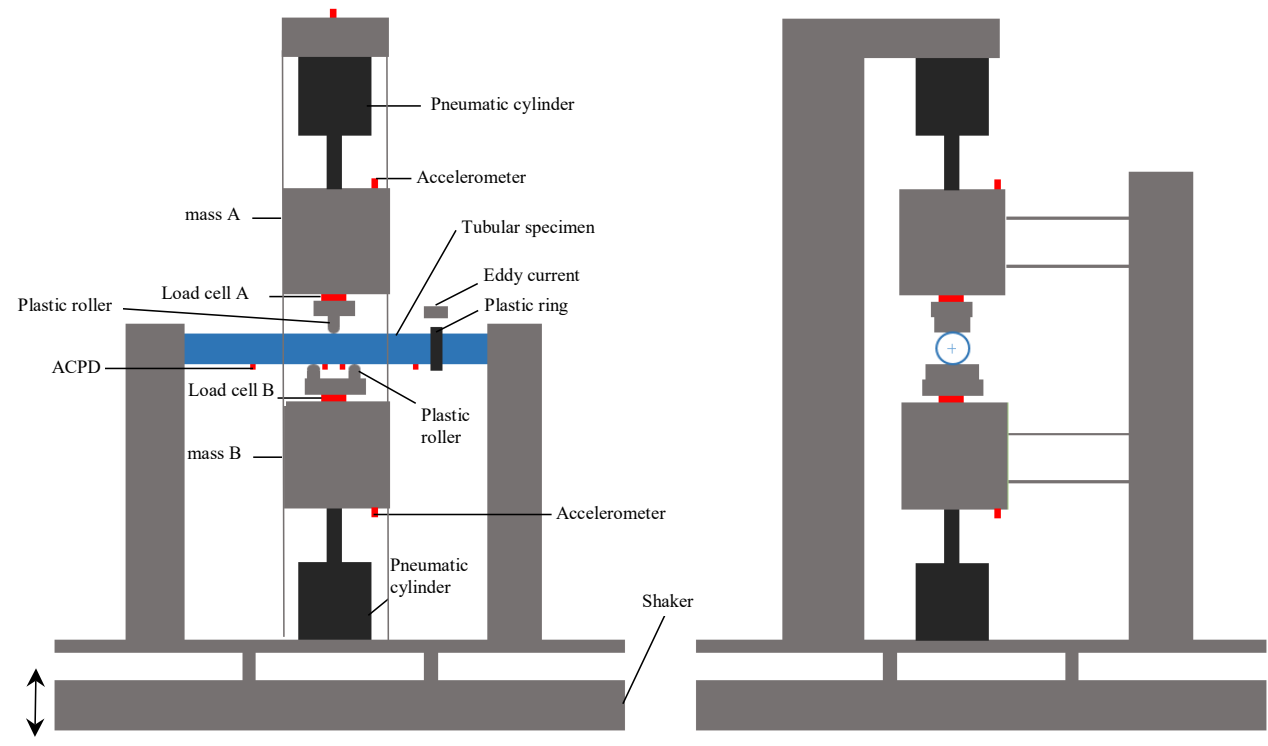

Fig. 1. Schematic diagram of the modified experimental fatigue rig. The electromechanical shaker provides a base excitation to the experimental rig so that cyclic stresses in the tubular specimen and initiate and propagate cracks. Two force sensors and three accelerometers combined with the proximity sensor (eddy current) and alternating current potential difference (ACPD) measurement setup are shown

Flexible specimen jaws have been designed to accommodate a range of specimen sizes and shapes including tubular. Different pipe sections can be mounted to the rig by adjusting the sliding jaws. The sliding jaws are mechanically fastened to the specimen support via bolts. To apply the loads directly to the specimen, high strength plastic has been used for the rollers to minimise the damping at the load interface. The plastic type is cast nylon 6 with hardness of 170 ball indentation with high resistance to abrasion. The white plastic rollers can be seen in Fig. 1 and Fig. 2(c).

As part of the modifications to the rig two force sensors were designed for use between the masses (A and B) and the specimen. These two force load cells were designed to be located as close as possible to the specimen to obtain a better reading of the applied forces on the specimen. The location of load 
cell A and load cell B are shown in Fig. 1, Fig. 2(b) and Fig. 2(c). The vertical displacement of the tubular specimen is monitored by the eddy current probe. The eddy current probe is facing a flat metal plate attached to a plastic ring under the eddy current probe for the deflection measurement. The plastic ring and the flat plate is designed to enable eddy current reading in tubular specimens. The nonlinearity of the specimen displacement under force which is caused by the crack can be monitored from the eddy current prob. The crack propagation during the experiment is monitored using an ACPD (Alternative Current Potential Drop) device. Four ACPD cables, two for current and two for voltage, are screwed and tightened to the specimen. As part of the modification the ACPD cables where changed to solid core (single strand) wires to minimise the noise in the recorded data. In addition, a stand was designed and fitted to the rig to fix the wire slots. The accelerations of mass A, mass B and the base are recorded using the three accelerometers connected to them similar to [1].

(a)

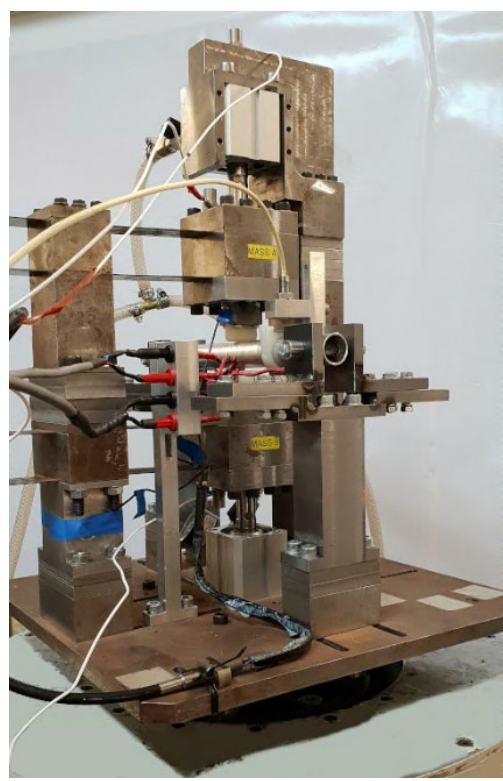

(b)

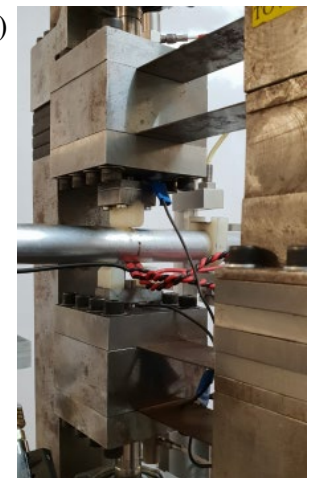

(c)

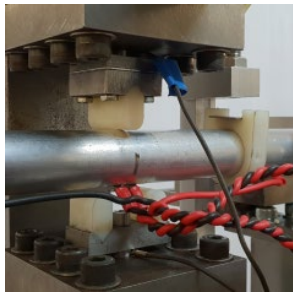

(d)

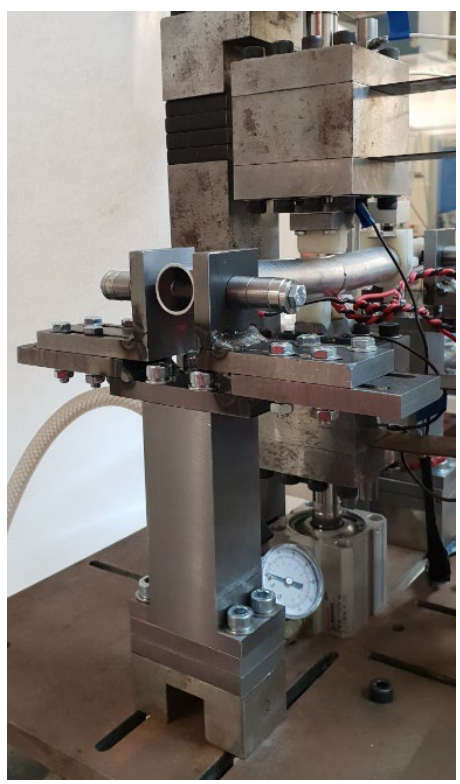

Fig. 2. (a) Photograph of the experimental fatigue rig on an electromechanical shaker; (b) close-up photograph of Masses A and B at top and bottom of the tubular specimen respectively, which are connected to a vertical tower fixture using leaf springs; (c) plastic rollers used to apply forces to the tubular specimen while the half circumference $\mathrm{v}$-shaped groove guides the direction of formation of the initial crack. The ACPD cables are attached close to the sides of groove to monitor crack length; (d) flexible jaws to facilitate experimentation on tubular specimens as well as arbitrary geometries

\subsection{Instrumentation and data acquisition}

Force sensors were calibrated using a $3 \mathrm{kN}$ Hounsfield load cell device in the materials laboratory at the University of Aberdeen. To investigate the relation between the ACPD machine reading and the crack depth, artificial cracks have been made into a tubular specimen. The results showed an acceptable linear correlation between the ACPD reading and crack depth for a tubular member. Force sensors measured force exerted on masses both above and below the specimen. The movement of fatigue rig was measured using an accelerometer and an eddy current probe detected the motions of the specimen. Wires were screwed to the specimen to measure fluctuations in voltage from an application of alternating current, so as to quantify crack length.

Fig. 3 shows the crack depths in 10 experimental tests and the correlation graphs for $30 \mathrm{~Hz}$ and $100 \mathrm{~Hz}$ current on a $25.4 \mathrm{~mm}$ diameter tube with wall thickness of $3.25 \mathrm{~mm}$. Similar studies such as [18] have shown an approximate linear relationship between the ACPD reading and the crack depth in the pipe specimens. The ACPD signal frequency was set to $30 \mathrm{~Hz}$ as well as $100 \mathrm{~Hz}$. 

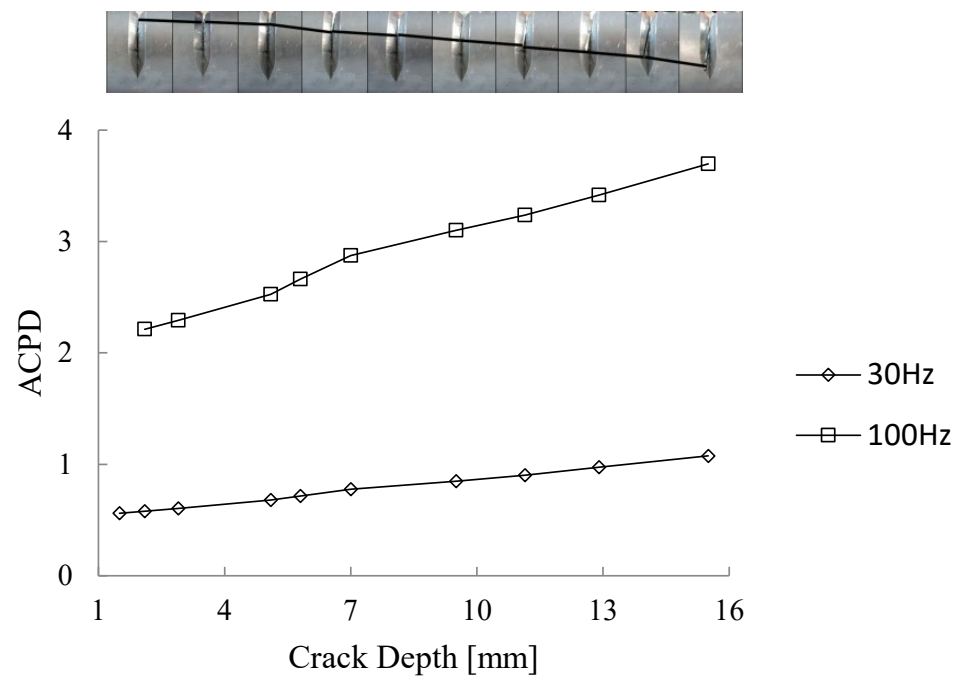

Fig. 3. ACPD voltage readings were plotted against crack depth in 10 experimental tests for a current passing through the crack at $30 \mathrm{~Hz}$ (diamond symbols) as well as $100 \mathrm{~Hz}$ (square symbols). Cracks were initiated and propagated in each of the 10 tests. The photographs from the 10 experiments are shown above the graph. The crack tips in the 10 photographs are connected with lines to show the trend of crack propagation

The leaf springs stiffness $(k)$ and damping ratio $(\zeta)$ were calculated by observing free damped vibration of masses A and B and record of the accelerations in 40 seconds. A total of fourteen free vibration experiments were conducted for mass A and mass B. Values of $14.181 \mathrm{kN} / \mathrm{m}$ for the stiffness of leaf spring A, 0.00188 for the damping ratio of leaf spring A, $14.392 \mathrm{kN} / \mathrm{m}$ for the stiffness of leaf spring B and 0.00141 for the damping ratio of leaf spring B were calculated.

The proximity sensor was calibrated using a displacement gauge. The displacement gauge has an accuracy of 0.001 inches and is placed just under the specimen to measure the vertical deflection of the specimen and calibrate the eddy current sensor. The data acquisition is performed using a new Labview program which was specifically developed for the modified fatigue rig. Data is acquired from seven sensors including three acceleration sensors, two force sensors, eddy current probe and ACPD.

\subsection{Details of tubular specimens}

Most fatigue experiments are carried out using American Society for Testing and Materials (ASTM) recommended specimen dimensions which may not necessarily be similar to real structural element. Fatigue experiments on non-conventional ASTM tubular specimens is subject of recent research such as the study by Fatemi et al. [19] in which new specimens for fatigue testing were introduced and utilised. Fischer et al. [20] performed fatigue experiment on cracked round bar using linear-torsion electro-dynamic test machine. Longitudinal grooves with different depths and shapes were used in [21] for fatigue experiments.

In this experiment, an extrusion pipe section made of aluminium alloy 6082-T6 with outer diameter of $25.4 \mathrm{~mm}$ and thickness of $1.626 \mathrm{~mm}$ was selected for the experiments. Fatigue experiments on aluminium alloy 6082-T6 is used in a number of studies such as [22] and [23]. To induce stress concentration and influence the orientation of the crack, notches can be introduced in fatigue specimens. A half circumference v-groove was made on the pipe specimen to affect the desired direction of crack propagation. The v-groove in pipe specimens are used in some research work such as [24] to study the fatigue on the pipe sections. A total of fifteen specimens were produced in the workshop covering three groove depths of $1 \mathrm{~mm}, 1.2 \mathrm{~mm}$ and $1.3 \mathrm{~mm}$. The corner of the groove has a $0.1 \mathrm{~mm}$ fillet radius. The specimens are $300 \mathrm{~mm}$ long with circular hole at one end and sliding hole at the other end. Side view of the specimen with section view at the centre of groove are shown in Appendix A. The details of fifteen specimens are shown in Tab. 1. 
Table 1. Experiment ID, specimen type and groove depth

\begin{tabular}{ccc}
\hline Experiment ID & Specimen Type & Groove Depth $(\mathrm{mm})$ \\
\hline $1-4$ and $13-15$ & SP1 & 1.0 \\
5-7 and 11-12 & SP2 & 1.2 \\
$8-10$ & SP3 & 1.3 \\
\hline
\end{tabular}

\subsection{Finite Element model}

Finite Element (FE) analysis was carried out to calculate stress concentration factors. The locations and directions of crack are known to occur at places of maximum principal stress. This matter also has experimentally been demonstrated in research such as [25].

A finite element model describing the geometry of each specimen was created in Abaqus software to calculate the stress concentration factor (SCF) at the groove location and work out the peak stress the specimen experiences during the loading. These would then form the basis of signal input to electromechanical shaker so as to mimic actual loading conditions in real life. Three-dimensional geometry specimens were created and solid reduced-integration elements C3D8R and C3D6 were used to mesh the geometry. Three distinct models were generated for the each of three geometry of specimens. A very refined mesh size was used at the groove location. The block of refined elements was connected to the rest of larger size elements using tie technique in Abaqus. Linear material was assigned to the elements and the peak principal stress was compared to nominal stress under bending to derive the stress concentration factor (SCF). Fig. 4 demonstrates the 3D finite element model of specimen SP1. The stress concentration factors from the finite element models are presented in Tab. 2.

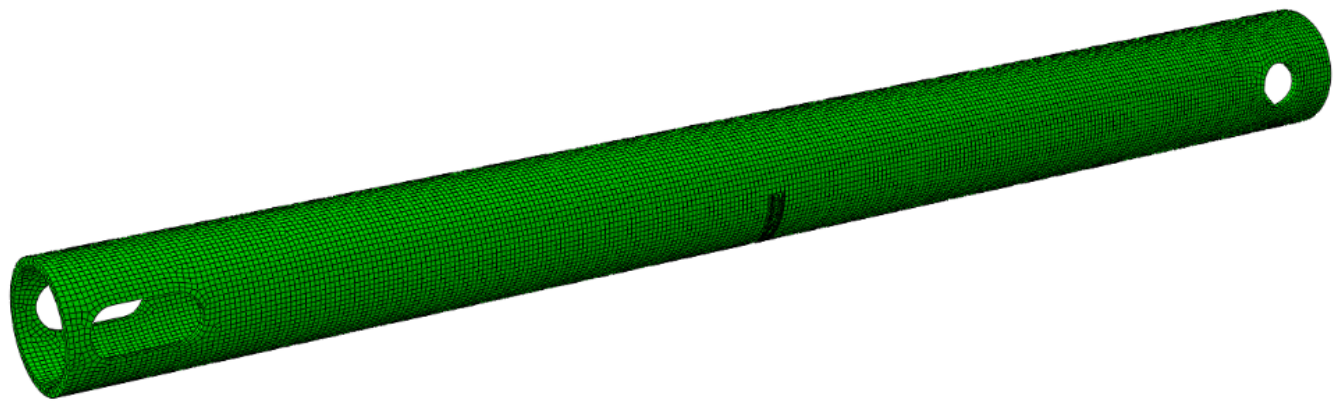

(a)

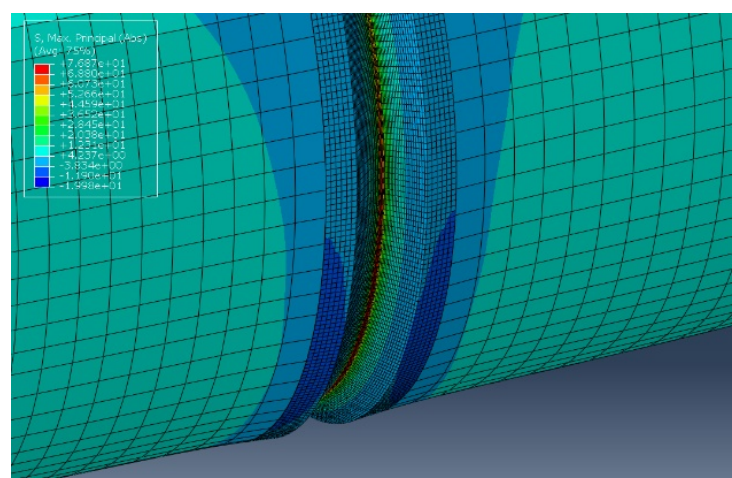

(b)

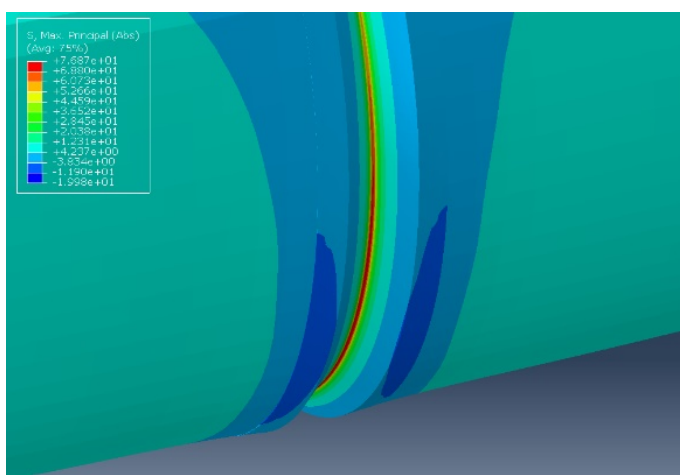

(c)

Fig. 4. (a) 3D (three-dimensional) global solid model of the cracked specimen in Abaqus software; (b) localised refined mesh for use in finite element analysis at groove in a tubular specimen, and (c) numerical computation of stress concentration (based on maximum principal stress) under unit load bending of the specimen 
Table 2. Stress Concentration Factor (SCF) as a function of the groove depth for the studied specimens (SP1, SP2 or SP3)

\begin{tabular}{ccc}
\hline Specimen & Groove Depth $(\mathrm{mm})$ & SCF \\
\hline SP1 & 1.0 & 3.50 \\
SP2 & 1.2 & 4.21 \\
SP3 & 1.3 & 3.84 \\
\hline
\end{tabular}

There is a peak value of stress at the bottom of the groove, and there is a heightened value of stress at the lower half of the tubular section. The stress concentration factors were used to calibrate the signal amplitude to the shaker and calculate the stress ranges in the specimen.

\section{EXPERIMENTAL RESULTS}

In this section the response of the fatigue rig under harmonic excitation is presented. Then the results of fatigue experiments for fifteen specimens are detailed including the accumulated fatigue damage and the comparison against design S-N curves from BS EN 1999-1-3 [26]. The specimen displacement from eddy current and comparison with elastic beam theory at time of crack initiation is also shown.

Depending to the number of cycles the fatigue S-N curves can be categorised as Low Cycle Fatigue (LCF) for less than $10^{5}$ cycles, High Cycle Fatigue (HCF) for above $10^{5}$ and below $10^{7}$, and Very High Cycle Fatigue (VHCF) for above $10^{7}$ cycles [27]. Other references such as BS EN 1999-1-3 [26] code suggests a threshold of $10^{5}$ cycles between low and high cycle fatigue.

\subsection{Harmonic test}

The modified fatigue rig was subject to harmonic excitation on an electromechanical shaker at a frequency of $20 \mathrm{~Hz}$. A signal generator specifies the required amplitude and frequency, based on stress concentration factors computed a priori. This acts as an input to the shaker so that it would move in

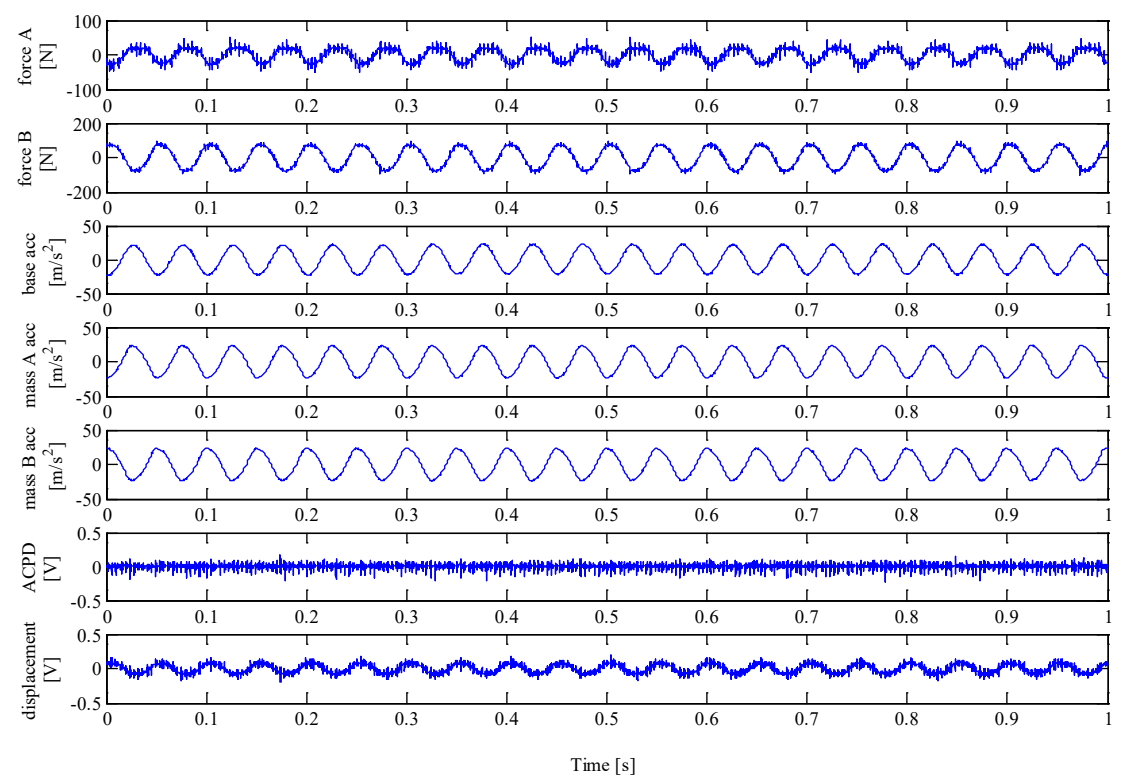

Fig. 5. Time histories captured by Labview for force on Mass A on top of the tubular specimen (first graph), force on Mass B below the tubular specimen (second graph from the top), acceleration of shaker table (third graph), acceleration of Mass A (fourth graph), acceleration of Mass B (fifth graph), voltage across crack (sixth graph) and voltage from eddy current probe measuring lateral oscillations of cracked specimen, when the shaker table oscillated harmonically at $20 \mathrm{~Hz}$ during one second 
accordance to it. Force, acceleration, and specimen displacement are monitored and measured. The Labview programme records data pertaining to seven parameters and converts the readings to appropriate units at a sampling rate of $2000 \mathrm{~Hz}$. System dynamic responses are shown in Fig. 5. The response from the two force sensors, the three accelerometers are sinusoidal and proportional to the excitation form the shaker. Similarly, the proximity sensor eddy current sensor shows a periodic deflection of the specimen. The ACPD reading fluctuates slightly while averaging over a constant value, indicating no crack formation or propagation in the one second of the measured data. Force B, which is the force on Mass B, is out of phase to Force A (the force on Mass A), and the acceleration of Mass B is asynchronous to that for Mass A. The displacement of the specimen is shown in the last graph of Fig. 5 at the bottom. While the specimen contributes to a stiffness characteristic, it also responds in accordance to the base excitation. There is electrical noise in some of the signals from measurement which perhaps could be improved further with shielding in future work.

\subsection{Fatigue experiments}

A total of fifteen fatigue experiments were performed and the results were post processed. As can be seen in Fig. 1 and Fig. 2 the force from Mass A is applied on the specimen via a single plastic roller while the force from Mass B is applied on the specimen via two plastic rollers. These two forces are available from the load cells data. The stress range at the groove location was calculated using the findings of the finite element analysis.

The loading frequency of the experiments varied from $15 \mathrm{~Hz}$ to $30 \mathrm{~Hz}$. The fatigue design relationship in BS EN 1999-1-3 [26] code in range between $10^{5}$ to $5 \times 10^{6}$ cycles is shown in Eq. (1). $\Delta \sigma_{c}$ is the reference value of fatigue strength at $2 \times 10^{6}$ cycles, depending on the detail category while $\Delta \sigma_{i}$ is the stress range for the principal stresses at the constructional detail. $m_{1}$ is the inverse slope of the $\Delta \sigma$-N curve, depending on the detail category. $\gamma_{F f}$ is the partial factor allowing for uncertainties in the loading spectrum and analysis of response and $\gamma_{M f}$ is the partial factor for uncertainties in materials and execution. The value of 1.0 for partial factors $\gamma_{F f}$ and $\gamma_{M f}$ offset the SN curves by a mean minus two standard deviation from experimental data in BS EN 1999-1-3 [26]. A value of 1.0 is recommended by BS EN 1999-1-3 for safe life design.

$$
N_{i}=2 \times 10^{6}\left[\frac{\Delta \sigma_{c}}{\Delta \sigma_{i}} \frac{1}{\gamma_{F f} \cdot \gamma_{M f}}\right]^{m_{1}} .
$$

In cases where a significant damage is caused by high stress ranges applied less than $10^{5}$ times, the nominal S-N curves can be unnecessarily be conservative [26]. For low cycle fatigue in the range of $10^{3}$ to $10^{5}$ cycles, the fatigue design relationship is defined by Eq. (2) in BS EN 1999-1-3 [26], in which $m_{0}$ is the inverse logarithmic slope of the $\Delta \sigma$-N curve in the range $10^{3}$ and $10^{5}$ cycles.

$$
N_{i}=\left[\frac{\Delta \sigma_{c}}{\Delta \sigma_{i}} \frac{1}{\gamma_{F f} \cdot \gamma_{M f}}\right]^{m_{0}} \times 20^{\frac{m_{0}}{m_{1}}} \times 10^{5} .
$$

Detail type 1.4 and category 71-7 are selected for the specimens in the experimental studies accordingly to BS EN 1999-1-3 [26]. This category covers surface irregularity in tubes and extrusions, which is directly applicable to v-grooves. As such, it is deemed the most suitable fit. The S-N curve from BS EN 1999-1-3 3 [26] for different details including detail category 71-7 is shown in Fig. 6. The S-N curve constants for category 71-7 are noted as $\Delta \sigma_{c}, m_{1}$ and $m_{0}$ with values of $71 \mathrm{~N} / \mathrm{mm}^{2}, 7$ and 4 respectively.

The fatigue S-N curves in BS EN 1999-1-3 [26] are based on a 95\% probability of survival [26,28]. The number of cycles $n_{i}$ is available from the data acquisition while $N_{i}$ is calculated from Eq. (1) or Eq. (2), whichever is applicable depending on definition of low cycle and high cycle fatigue. Total damage value $\left(D_{L}\right)$ is calculated for the full life and is defined as sum of $\frac{n_{i}}{N_{i}}$. In each experiment the value of $\frac{n_{i}}{N_{i}}$ was calculated for each cycle and summed up to work out the damage $D_{L}$. According to Palmgren-Miner's rule failure occurs once sum of $\frac{n_{i}}{N_{i}}$ are equal to 1.0 . 


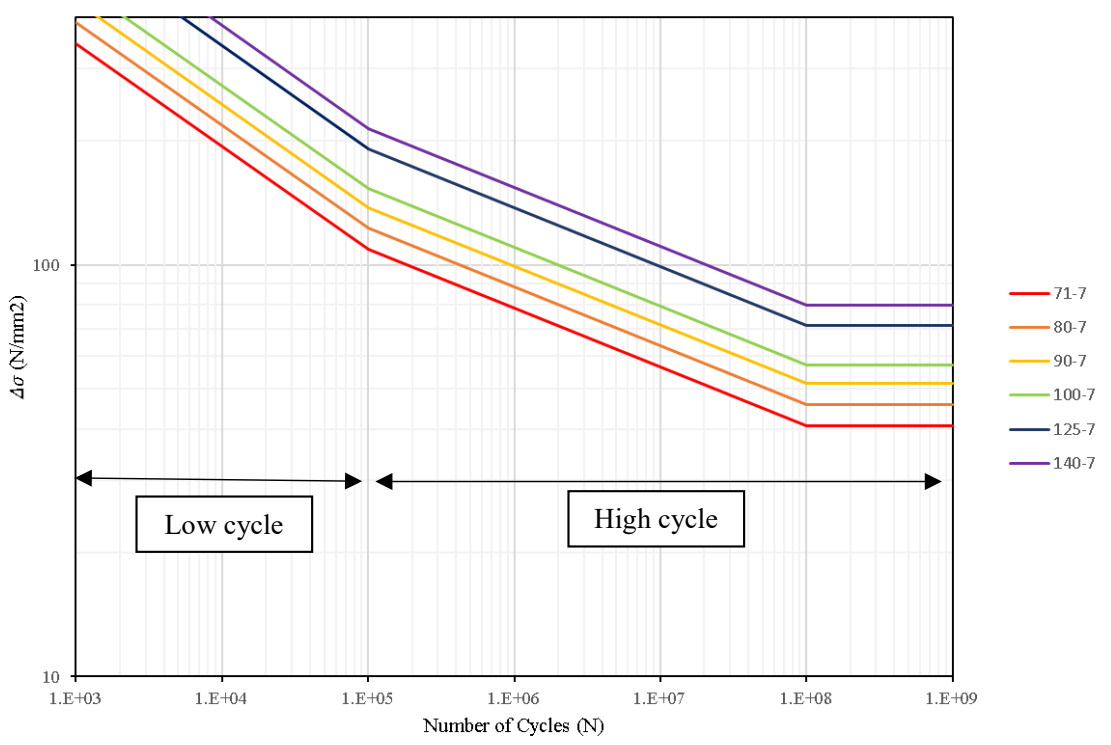

Fig. 6. BS EN 1999-1-3 [26] fatigue strength curves for detail categories 71-7, 80-7, 90-7, 100-7, 125-7 and 140-7. Detail category 71-7 pertains to product forms such as sheets, plates, extrusions, tubes and forgings such that there are surface notches on flat or tubular specimens. There is no requirement for a surface finish, which is applicable to the cracked specimens in this experiment

Ultimate achieved cycles for each experiment are shown in Tab. 3. The total damage combination, $D_{L}$, was calculated for each of fifteen experiments based on this data. The achieved $D_{L}$ exceeded the BS EN 1999-1-3 predicted a value of 1.0 in all fifteen experiments. This shows relatively high conservatism referring to aluminium code BS EN 1999-1-3 design S-N curves. In addition, the code recommends a value of 1.0 for partial factors $\gamma_{F f}$ and $\gamma_{M f}$. These point towards safe design and are likely to be very conservative and generic.

Table 3. Number of cycles to failure for each experiment corresponding to fifteen samples

\begin{tabular}{ccc}
\hline Experiment ID & Specimen Type & Number of cycles \\
\hline 1 & SP1 & 254212 \\
2 & SP1 & 543424 \\
3 & SP1 & 4043 \\
4 & SP1 & 125284 \\
5 & SP2 & 193653 \\
6 & SP2 & 86408 \\
7 & SP2 & 300115 \\
8 & SP3 & 245998 \\
9 & SP3 & 92692 \\
10 & SP3 & 223595 \\
11 & SP2a & 3096 \\
12 & SP2a & 103562 \\
13 & SP1a & 270358 \\
14 & SP1a & 63537 \\
15 & SP1a & 159459 \\
\hline
\end{tabular}

In further work based on periodic cyclic loading, Sun et al. in [29] performed statistical fatigue damage model under constant and variable loading according to Miner's damage criterion. This would have taken an arbitrary loading case into consideration. They concluded that the Miner's rule is statistically inconsistent. This experiment showed that the damage accumulation based on Miner's rule is 
not consistent. However, in all experiments the total damage was greater than 1.0, verifying the implicit conservatism in BS EN 1999-1-3 [26]. Alternative damage accumulation methods have been developed and proposed such as nonlinear damage accumulation proposed by Zhu et al. [30] or Dias et al. [31].

\subsection{Crack growth}

The crack propagation life of aluminium components is lower compared to steel due to the higher crack growth rate and the lower fracture toughness [28]. This phenomenon results in a rather small fatigue crack at the moment of fracture [28]. Matelect ACPD machine used to read the ACPD readings during the course of the experiments. As explained and demonstrated in Fig. 3 the crack depth and ACPD reading have a linear relationship. At each cycle of load the crack opens and closes. ACPD reaches maximum peak when the crack opens. The crack depth size at the end of each experiment was used to calibrate the ACPD readings for each experiment.

(b)

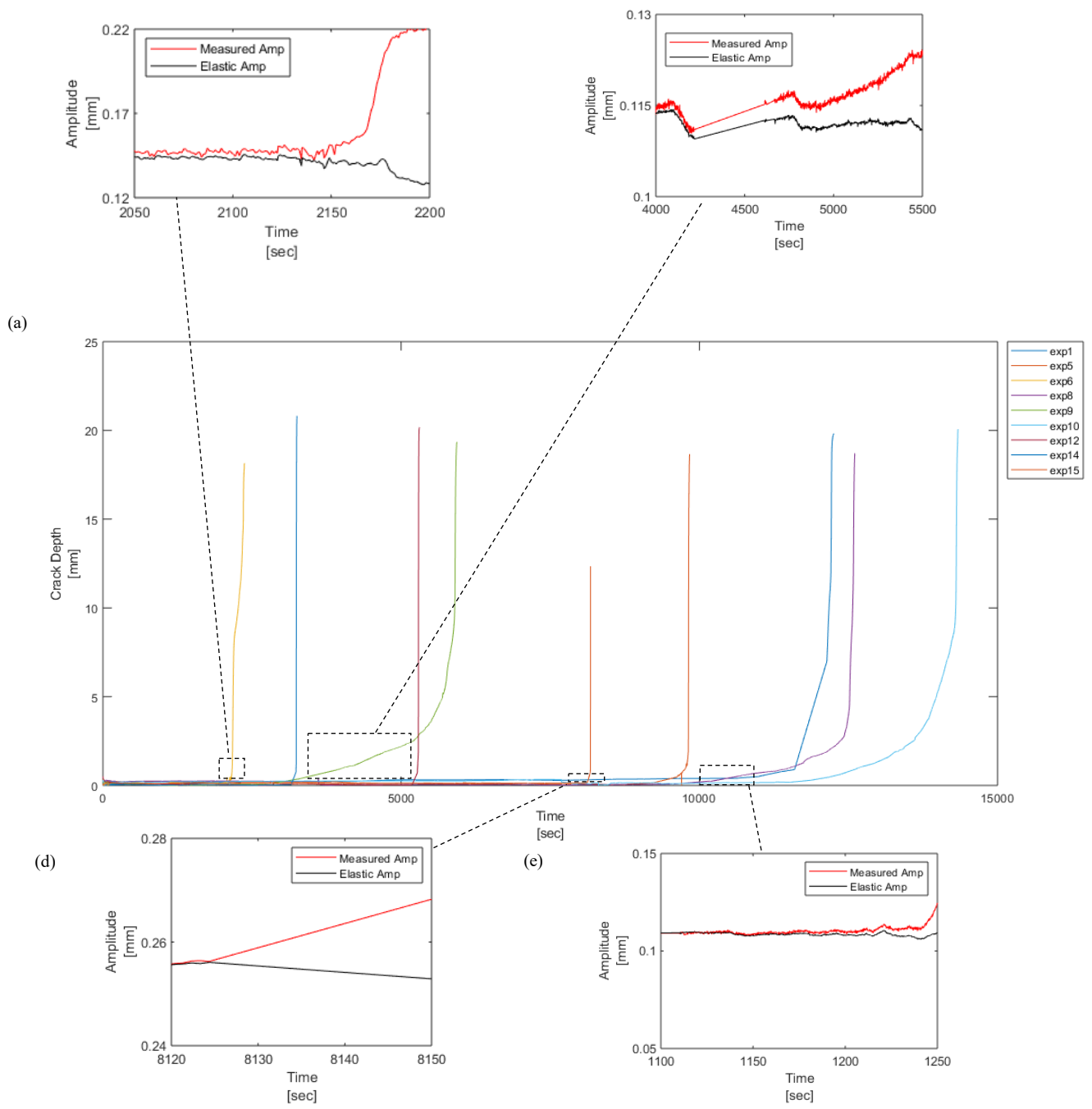

Fig. 7. (a) Plot of crack depth against time, of which the former was measured using the alternating current potential difference (ACPD) method. Each line is drawn with a different colour, and the legend corresponds to the experiment numbers listed in Tab. 3; (b) measured crack length (in red) and prediction of crack length from linear elastic theory (in black) plotted against time in Experiment 6; (c) measured crack length (in red) and prediction of crack length from linear elastic theory (in black) plotted against time in Experiment 9; (d) measured crack length (in red) and prediction of crack length from linear elastic theory (in black) in Experiment 15 and (e) measured crack length (in red) and prediction of crack length from linear elastic theory in Experiment 8 
Fig. 7(a) shows the crack growth in nine of the experiments. The ACPD reading was not available for 6 of the experiments due to the noise in the recorded data. The source of the noise could not be determined but anticipated to be from induction from other electrical equipment in the lab. As can be seen the crack is formed at the latter part of the experiments with fairly high propagation rate. The crack propagation rate is higher in SP1 specimens while SP3 specimens exhibit a lower crack propagation rate. The proximity sensor shows actual displacements of the specimen. The displacement range (twice the displacement amplitude) was compared against the elastic displacement calculated from recorded forces on the specimen. This comparison in shown in Fig. 7(b, c, d, e) for Experiments 6, 9, 15 and 8. The comparison highlights the deviation from elastic displacement to nonlinear due to formation of the crack and change of stiffness.

In Fig. 7(a), a recurring trend in these experiments was that the rate of crack growth increased significantly at specific instants in time, and this data would be useful in an analytical model of fatigue growth for future work. Figs. 7(b), 7(c), 7(d) and 7(e) highlight the nonlinear displacements of specimens from experimental measurement and the divergence at the crack formation point.

\section{CONCLUSIONS}

The main contribution from this work is the quantification of crack propagation in a specific geometry, namely tubular cross-sections. The specimen geometry was defined in finite element analysis so as to calculate stress concentration factors. These, in turn, facilitated an input signal to electromechanical shaker for subsequent experiments. The vibration, crack length and force are measured while time varies. These provide insight to the energy input mechanism and how it influences the rate of crack propagation in relation to system dynamic response.

The modified fatigue rig can be used to perform experiments on a variety of pipe sections to investigate the fatigue life of specimens and crack propagations. Designing and implementing two loadcells close to the specimen resulted in the direct measurement of the loads to derive the stress and stress range in the specimen and perform real condition fatigue testing. Finite element analysis was used to calculate the stress concentration factor and used to amplify the stress in the calculations. Total damage was summed up in each experiment. A total 15 experiments with 3 specimen types were performed. The results showed that in all 15 experiments, the calculated total damage exceeded BS EN 1999-1-3 [26] predictions, indicating the conservatism in design S-N curves. Groove depths of $1.0 \mathrm{~mm}, 1.2 \mathrm{~mm}$ and $1.3 \mathrm{~mm}$ have been investigated.

The new fatigue rig enabled investigating the fatigue behaviour of the tubular specimens and performance of the cracked specimens. Harmonic and stochastic loading can also be applied in the tests. Different stress raisers such as grooves and holes on the tube specimens can be tested. Stress concentration factors of 3.50, 4.21 and 3.84 form the basis for signal input to an electromechanical shaker and calculation of the stress range for fatigue life prediction.

The available S-N curves in the codes such as BS EN 1999-1-3 [26] can be generic and the partial factors embedded in the codes to offset the S-N curves for design are broad with limited guidance for refinement. The comparison between the calculated fatigue life from the S-N curves and the experiment can potentially lead to develop new S-N curves for specific cases which are tailored for a configuration of interest. In addition the damage accumulation according to Miner's rule shown to be conservative when used in conjunction with S-N curves in BS EN 1999-1-3 [26]. The newly developed fatigue rig can be used to perform experiments on specimens with real details which result in modified and fit for purpose S-N curves.

The ACPD readings enabled direct monitoring of the crack propagation in experiments. Comparison of elastic displacement and the records from ACPD allows locating the transition from linear elastic to nonlinear phase, indicating the time in which a crack is formed. The onset of crack propagation is observed from experimental time histories. The fatigue rig can be used for random and impact loading in future. For this purpose, a signal generation activity is needed to define the required signals to excite the shaker. 


\section{REFERENCES}

[1] C. H. Foong, M. Wiercigroch, and W. F. Deans. Novel dynamic fatigue-testing device: design and measurements. Measurement Science and Technology, 17, (8), (2006), pp. 2218-2226. https://doi.org/10.1088/0957$0233 / 17 / 8 / 023$.

[2] N. Jakšić, C. H. Foong, M. Wiercigroch, and M. Boltežar. Parameter identification of the fatigue-testing rig. International Journal of Mechanical Sciences, 50, (7), (2008), pp. 1142-1152. https://doi.org/10.1016/j.ijmecsci.2008.04.007.

[3] Fatigue design of offshore steel structures. DNV Recommended Practice DNV-RP-C203, (2016).

[4] J. A. Ronevich, E. J. Song, Z. Feng, Y. Wang, C. D'Elia, and M. R. Hill. Fatigue crack growth rates in high pressure hydrogen gas for multiple X100 pipeline welds accounting for crack location and residual stress. Engineering Fracture Mechanics, 228, (2020). https://doi.org/10.1016/j.engfracmech.2019.106846.

[5] M. J. Gam, B. S. Jang, and J. H. Park. A study on the fatigue analysis for a vertical caisson on FPSO subjected to the nonlinear wave loading. Ocean Engineering, 137, (2017), pp. 151-165. https://doi.org/10.1016/j.oceaneng.2017.03.057.

[6] R. Shoghi and H. Shiri. Re-assessment of trench effect on fatigue performance of steel catenary risers in the touchdown zone. Applied Ocean Research, 94, (2020). https://doi.org/10.1016/j.apor.2019.101989.

[7] L. Carneiro, X. Wang, and Y. Jiang. Cyclic deformation and fatigue behavior of 316L stainless steel processed by surface mechanical rolling treatment. International Journal of Fatigue, 134, (2020). https://doi.org/10.1016/j.ijfatigue.2019.105469.

[8] P. Xiang, L. J. Jia, M. Shi, and M. Wu. Ultra-low cycle fatigue life of aluminum alloy and its prediction using monotonic tension test results. Engineering Fracture Mechanics, 186, (2017), pp. 449-465. https://doi.org/10.1016/j.engfracmech.2017.11.006.

[9] W. Li, X. Xing, N. Gao, and P. Wang. Subsurface crack nucleation and growth behavior and energy-based life prediction of a titanium alloy in high-cycle and very-high-cycle regimes. Engineering Fracture Mechanics, 221, (2019). https://doi.org/10.1016/j.engfracmech.2019.106705.

[10] C. P. Okeke, A. N. Thite, J. F. Durodola, and M. T. Greenrod. A novel test rig for measuring bending fatigue using resonant behaviour. Procedia Structural Integrity, 13, (2018), pp. 1470-1475. https://doi.org/10.1016/j.prostr.2018.12.303.

[11] N. Nagabhooshanam, S. Baskar, and P. K. Nagarajan. Design and fabrication of fatigue test rig and preliminary investigation on flax composite beam. Materials Today: Proceedings, 5, (5), (2018), pp. 11771-11779. https://doi.org/10.1016/j.matpr.2018.02.146.

[12] H. Wei, P. Carrion, J. Chen, A. Imanian, N. Shamsaei, N. Iyyer, and Y. Liu. Multiaxial high-cycle fatigue life prediction under random spectrum loadings. International Journal of Fatigue, 134, (2020). https://doi.org/10.1016/j.ijfatigue.2019.105462.

[13] C. H. Foong, M. Wiercigroch, E. Pavlovskaia, and W. F. Deans. Nonlinear vibration caused by fatigue. Journal of Sound and Vibration, 303, (1-2), (2007), pp. 58-77. https://doi.org/10.1016/j.jsv.2006.12.008.

[14] C. H. Foong, E. Pavlovskaia, M. Wiercigroch, and W. F. Deans. Chaos caused by fatigue crack growth. Chaos, Solitons \& Fractals, 16, (5), (2003), pp. 651-659. https://doi.org/10.1016/s0960-0779(02)00449-6.

[15] T. Li, S. T. Lie, and Y. B. Shao. Fatigue and fracture strength of a multi-planar circular hollow section TT-joint. Journal of Constructional Steel Research, 129, (2017), pp. 101-110. https://doi.org/10.1016/j.jcsr.2016.11.001.

[16] P. Arora, P. K. Singh, V. Bhasin, K. K. Vaze, A. K. Ghosh, D. M. Pukazhendhi, P. Gandhi, and G. Raghava. Predictions for fatigue crack growth life of cracked pipes and pipe welds using RMS SIF approach and experimental validation. International Journal of Pressure Vessels and Piping, 88, (10), (2011), pp. 384-394. https://doi.org/10.1016/j.ijpvp.2011.07.003.

[17] Z. Mikulski and T. Lassen. Fatigue crack initiation and subsequent crack growth in fillet welded steel joints. International Journal of Fatigue, 120, (2019), pp. 303-318. https://doi.org/10.1016/j.ijfatigue.2018.11.014.

[18] P. Arora, P. K. Singh, V. Bhasin, K. K. Vaze, D. M. Pukazhendhi, P. Gandhi, and G. Raghava. Fatigue crack growth behavior in pipes and elbows of carbon steel and stainless steel materials. Procedia Engineering, 55, (2013), pp. 703-709. https://doi.org/10.1016/j.proeng.2013.03.318.

[19] A. Fatemi and R. Molaei. Novel specimen geometries for fatigue testing of additive manufactured metals under axial, torsion, and combined axial-torsion loadings. International Journal of Fatigue, 130, (2020). https://doi.org/10.1016/j.ijfatigue.2019.105287.

[20] J. Fischer, P. J. Freudenthaler, P. R. Bradler, and R. W. Lang. Novel test system and test procedure for fatigue crack growth testing with cracked round bar (CRB) specimens. Polymer Testing, 78, (2019), p. 105998. https://doi.org/10.1016/j.polymertesting.2019.105998.

[21] X. Zheng, X. Zhang, L. Ma, W. Wang, and J. Yu. Mechanical characterization of notched high density polyethylene (HDPE) pipe: Testing and prediction. International Journal of Pressure Vessels and Piping, 173, (2019), pp. 1119. https://doi.org/10.1016/j.ijpvp.2019.04.016. 
[22] D. Jíša, P. Liškutín, T. Kruml, and J. Polák. Small fatigue crack growth in aluminium alloy EN-AW $6082 / T 6$. International Journal of Fatigue, 32, (12), (2010), pp. 1913-1920. https://doi.org/10.1016/j.ijfatigue.2010.06.003.

[23] K. Kluger. Fatigue life estimation for 2017A-T4 and 6082-T6 aluminium alloys subjected to bending-torsion with mean stress. International Journal of Fatigue, 80, (2015), pp. 22-29. https://doi.org/10.1016/j.ijfatigue.2015.05.005.

[24] V. L. Neelakantha, T. Jayaraju, P. Naik, D. Kumar, and C. R. Rajashekar. Determination of fracture toughness and fatigue crack growth rate using circumferentially cracked round bar specimens of Al2014T651. Aerospace Science and Technology, 47, (2015), pp. 92-97. https://doi.org/10.1016/j.ast.2015.09.023.

[25] V. Chaves, G. Beretta, J. A. Balbín, and A. Navarro. Fatigue life and crack growth direction in 7075-T6 aluminium alloy specimens with a circular hole under biaxial loading. International Journal of Fatigue, 125, (2019), pp. 222-236. https://doi.org/10.1016/j.ijfatigue.2019.03.031.

[26] BS EN 1999-1-3:2007. Eurocode 9: Design of aluminum structures, Part 1-3: Structures susceptible to fatigue. British Standard.

[27] P. Schaumann and S. Steppeler. Fatigue tests of axially loaded butt welds up to very high cycles. Procedia Engineering, 66, (2013), pp. 88-97. https://doi.org/10.1016/j.proeng.2013.12.065.

[28] J. Maljaars, M. Lukić, and F. Soetens. Comparison between the Eurocode for fatigue of steel structures, EN 1993-1-9, and the Eurocode for fatigue of aluminium structures, EN 1999-1-3. In 5th International Conference on Fatigue Design, Elsevier, (2013), pp. 34-48.

[29] Q. Sun, H. N. Dui, and X. L. Fan. A statistically consistent fatigue damage model based on Miner's rule. International Journal of Fatigue, 69, (2014), pp. 16-21. https://doi.org/10.1016/j.ijfatigue.2013.04.006.

[30] S. P. Zhu, D. Liao, Q. Liu, J. A. F. O. Correia, and A. M. P. De Jesus. Nonlinear fatigue damage accumulation: isodamage curve-based model and life prediction aspects. International Journal of Fatigue, 128, (2019). https://doi.org/10.1016/j.ijfatigue.2019.105185.

[31] J. P. Dias, S. Ekwaro Osire, A. Cunha Jr, S. Dabetwar, A. Nispel, F. M. Alemayehu, and H. B. Endeshaw. Parametric probabilistic approach for cumulative fatigue damage using double linear damage rule considering limited data. International Journal of Fatigue, 127, (2019), pp. 246-258. https://doi.org/10.1016/j.ijfatigue.2019.06.011.

\section{APPENDIX A.}

In this appendix the geometry of the specimen is shown.

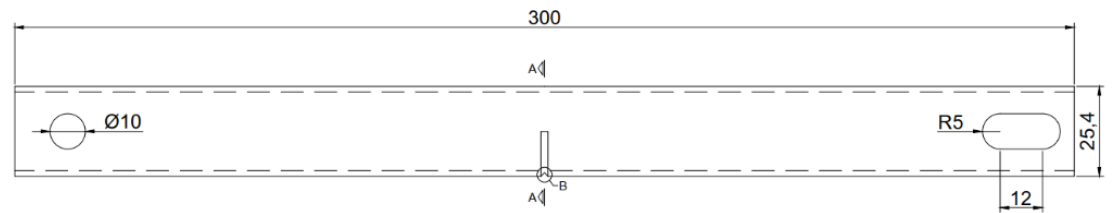

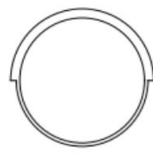

Section A-A

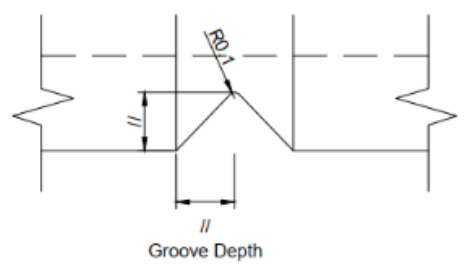

Detail B

Fig. A1. Geometry of the aluminium specimen used in experiments 